\title{
SOLUTION POLYMERIZATION OF METHYL-METHACRYLATE AT HIGH CONVERSION IN A RECYCLE TUBULAR REACTOR
}

\author{
P.-A.Fleury, Th.Meyer, A.Renken \\ Institut de génie chimique, Ecole Polytechnique Fédérale (EPFL) \\ CH-1015 Lausanne, Switzerland
}

\begin{abstract}
The kinetics of the solution polymerization of methyl-methacrylate is characterized by a strong increase of viscosity up to 6 orders of magnitude and auto-acceleration of the reaction rate due to the gel-effect. This can lead to thermal and kinetic reactor instabilities. The kinetics were determined separately using a differential scanning calorimeter and described with a modified published model. The model predictions are verified in pilot plant experiments in a temperature range from $130-170^{\circ} \mathrm{C}$.
\end{abstract}

\section{KEYWORDS}

Methyl-methacrylate, polymerization kinetics, tubular reactor, static mixer, reactor modelling

\section{INTRODUCTION}

In previous work on radical bulk polymerization in static mixers it was shown, that radial mixing in the tube is quite effective and axial dispersion can be neglected even at high monomer conversion resulting in large viscosity gradients. The proposed reactor concept consisting of a loop followed by a tubular reactor to complete conversion (Nguyen et al.,1985), was proved on industrial scale for relatively slow polymerization processes as the thermal bulk polymerization of styrene (Sulzer,1991a). The aim of the present study is to get experimental information on the reactor behavior at high heat load. For this purpose the continuous radical polymerization of methyl-methacrylate (MMA) was chosen as model reaction. At temperatures higher than $130^{\circ} \mathrm{C}$ the reaction rate is high enough to get conversions of more than $90 \%$ at mean residence times of about 10 min leading to a specific heat production of more than $1 \mathrm{MW} / \mathrm{m}^{3}$. Depending on the mixer type used temperature differences between wall and bulk are in the range of $15-30^{\circ} \mathrm{C}$.

\section{POLYMERIZATION MODEL}

The kinetics of the solution polymerization was studied in independent experiences using differential scanning calorimetry (DSC). ( Malavasic et al.,1984, Alberda and Tan, 1988). In order to evaluate the measured thermogram correctly, it is necessary to follow the polymerization reaction until no heat evolution can be detected and subsequently to run the reaction to complete conversion. The general kinetic model for the free radical polymerization assuming quasi steady state can be expressed as follows:

$$
-R_{M}=R_{P}=k_{p o} \cdot f_{p} \sqrt{2 f_{a} k_{d} C_{I} f_{t} / k_{t o}} \cdot C_{M}
$$

where $k_{p o}$ and $k_{t o}$ are the propagation and termination rate constants at very low conversion and $f_{p_{t}}, f_{t}$ the correction functions depending on temperature, polymer concentration, solvent and initiator. They reflect the fact, that both $k_{p}$ and $k_{t}$ fall due to strong diffusion limitations as the solution becomes very 
viscous. This phenomena leads to a rate acceleration at high conversion and is known as gel- or Trommsdorff-effect. The gel-effect was studied experimentally as well as theoretically by a number of authors over the last 20 years. However, most of the studies of bulk polymerization have very often ignored the influence of solvents on $f_{p}, f_{t}$ and are carried out at temperatures lower than $100^{\circ} \mathrm{C}$. An exception is the PhD-study of Corpart-Monti (1990).

Kinetic experiments were conducted in the following parameter range:

$\begin{array}{lcr}\text { Temperature: } & 130-170 & { }^{\circ} \mathrm{C} \\ \text { Initiator concentration (DTBP): } & 0.0022-0.2 & \mathrm{kmol} / \mathrm{m}^{3} \\ \text { Mass fraction of solvent (ethylbenzene): } & 0.08-0.165 & -\end{array}$

The temperature range is above the glass transition of PMMA and therefore $k_{p}$ is not affected by the conversion increase $\left(f_{p}=1\right)$. In order to represent accurately the gel-effect one can choose between several expressions for $f_{t}$ suggested in literature. An example for a correction function based on the free volume theory was proposed by Balke and Hamielec (1973) and Marten and Hamielec (1979), modified by Ross and Laurence(1976) as well as by Stickler et al.(1984). The common feature of these models is the fact, that the termination rate is not or only slightly affected up to a critical free volume corresponding to monomer conversions. For higher conversions the termination rate is strongly influenced by the diffusion of polymer radicals and $k_{t}$ decreases exponentially. Models based on the free volume theory are characterized by a discontinuity in the correction function.

A continuous function to describe the gel-effect was proposed by Chiu et al.(1983) and Louie et al.(1985) considering the influence of diffusion on the termination process from the very beginning of the polymerization. Therefore the overall rate of termination decreases gradually with conversion and diffusion becomes dominant around certain conversion levels traditionally associated with the onset of the gel-effect. The model can be summarized as follows:

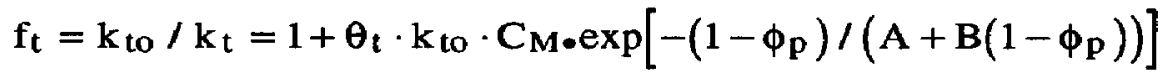

where $\theta_{t}, A, B$ are model parameters depending on temperature, initiator and solvent.

To fit the actual experimental data the presented model with three parameters is not easy to handle. In particular if a quasi steady state can not be assumed and at least three differential equations have to be resolved simultaneously. Therefore based on the general shape of the known correction functions an empirical relationship is proposed to describe the decrease of $k_{t}$ with increasing conversion

$$
\mathrm{f}_{\mathrm{t}}=\frac{\mathrm{k}_{\mathrm{to}}}{\mathrm{kt}}=1+\frac{\theta \cdot \mathrm{k}_{\mathrm{to}}}{\exp (\alpha-\beta \cdot \mathrm{X})}, \text { with } \theta=1(\mathrm{kmol} \mathrm{s}) / \mathrm{m}^{3}
$$

where $\alpha$ and $\beta$ are functions of temperature, solvent and initiator concentrations.

In Fig. 1 and 2 experimental results are compared with model calculations based on published kinetic constants for the MMA polymerization (Brandup and Immergut,1975; Louie et al.,1985) and adapting the model parameters $\alpha$ and $\beta$. Except for the very beginning the simulated curves are nearly identic to the experimental ones.

From the model calculations, the dependence of the parameters as function of initiator-, solventconcentration and of the reactor temperature could be determined. They are valid for the above mentioned experimental range.

$$
\alpha=23.15-12.50 \phi_{\text {so }} \quad \text { (4) } \quad \beta=29.848-37.25 \phi_{\text {so }}-6.966 C_{\text {Io }}-0.11(\mathrm{~T}-273)
$$

\section{PILOT PLANT STUDIES}

The pilot plant polymerization reactor is shown schematically in Fig.3. It consists of a recycle tubular reactor, in series with a tubular reactor and is constructed entirely of stainless steel 316 . The tubular part of the plant has an inner diameter of $20 \mathrm{~mm}$ and a total length of $1500 \mathrm{~mm}$. The recycle reactor consists of SMXL-mixers (Sulzer,1991a) with a total length of $2000 \mathrm{~mm}$ and $20 \mathrm{~mm}$ inner diameter. Flaschel et al. (1985) and Juvet (1989) determined axial mixing in Sulzer static mixers in the range of $10^{-4}<\operatorname{Re} 10^{4}$. They found for SMX and SMXL mixers residence time distributions corresponding to 40 and 30 ideal mixed cells in series respectively. Therefore plug flow can be considered under actual experimental conditions. The reactor is equipped with temperature and pressure indicators to determine axial temperature and pressure profiles. The mean residence time and the residence time distribution are measured regularly by dye tracer experiments to control the reactor behavior and to detect an eventual plugging of the tubes. In the present study only the recycle reactor is considered. 


\begin{tabular}{|c|c|c|c|c|c|c|}
\hline Run Nb & $\mathbf{W}_{\text {SO }}$ & $\tau$ & $\Phi$ & $\mathbf{T}_{\mathbf{w}}$ & $\Delta \mathbf{p}$ & $\mathbf{q}_{\mathbf{r}}$ \\
\hline$[-]$ & {$[-]$} & {$[\mathbf{m i n}]$} & {$[-]$} & {$\left[{ }^{\circ} \mathbf{C}\right]$} & {$[$ bar $]$} & {$\left[\mathbf{k W} / \mathbf{m}^{3}\right]$} \\
\hline 1 & 0.073 & 7.3 & 13.7 & 156.5 & 12.4 & 1057 \\
\hline 2 & 0.073 & 7.7 & 14.5 & 149.5 & 37.6 & 1123 \\
\hline 3 & 0.073 & 6.8 & 12.7 & 160.5 & 5.8 & 1079 \\
\hline 4 & 0.073 & 8.7 & 16.4 & 160.5 & 9.4 & 900 \\
\hline 5 & 0.165 & 41.5 & 32.2 & 140.0 & 18.6 & 188 \\
\hline 6 & 0.165 & 38.6 & 30.0 & 139.0 & 19.8 & 195 \\
\hline
\end{tabular}

Table 1 : Experimental conditions for pilot experiments.

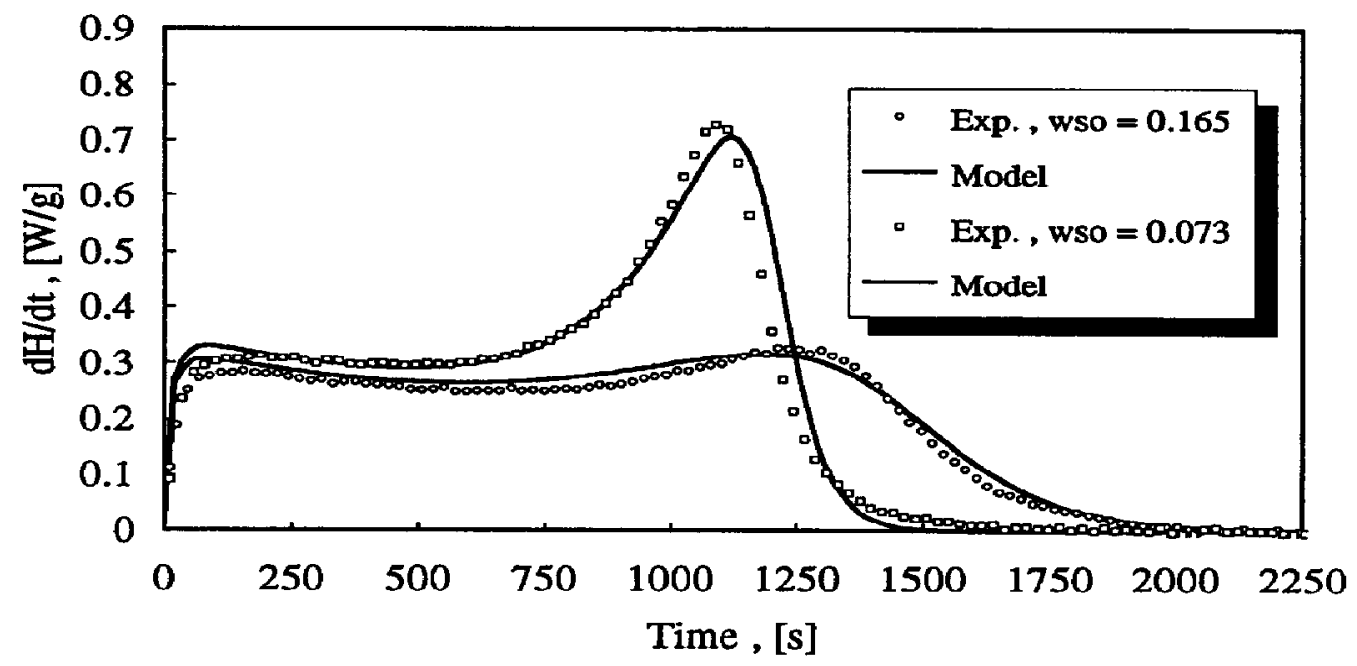

Fig 1 : Comparison of DSC measurements and simulated data for various solvent concentrations, $\mathrm{T}=135^{\circ} \mathrm{C}$.

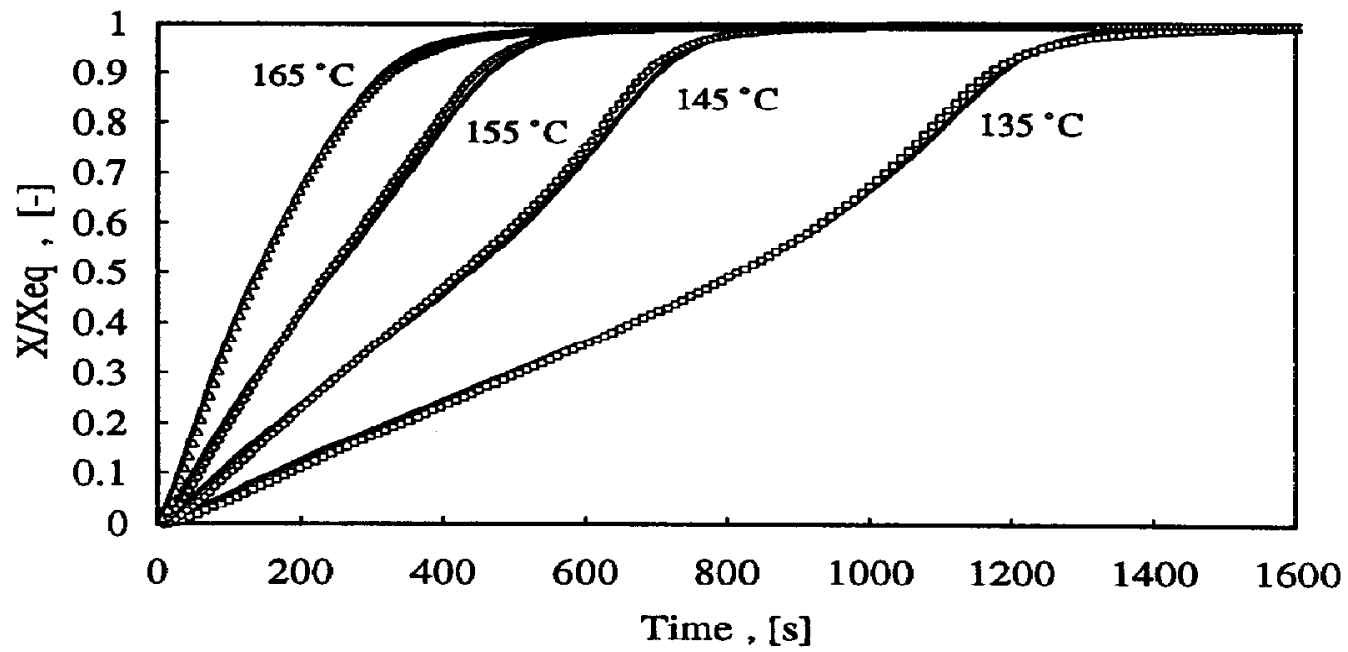

Fig 2 : Normalized conversion to the equilibrium vs time for DSC measurements and simulated data for various temperatures. 
Concentration and temperature profiles were calculated based on plug flow assumption:

Initiator conversion: $\quad \frac{d X_{I}}{d Z}=-\frac{R_{d}}{C_{I o}} \cdot \frac{\tau}{(1+\varphi)}$

Monomer conversion: $\quad \frac{\mathrm{dX}}{\mathrm{dZ}}=-\frac{\mathrm{R}_{\mathrm{M}}}{\mathrm{C}_{\mathrm{Mo}}} \cdot \frac{\tau}{(1+\varphi)}$

Temperature profile: $\quad \frac{d T}{d Z}=\frac{\tau}{\rho_{o} c_{p}(1+\varphi)}\left[\frac{4 h}{d_{t}}\left(T_{w}-T\right)+\left(-R_{M}\right)\left(-\Delta H_{r}\right)+\frac{\Delta p}{\rho c_{p}}\right]$

Density and thermal conductivity of the reaction mixture are dependent on solvent fraction, monomer conversion and temperature and were calculated simultaneously for any reactor position based on published data (Louie et al., 1985).

Due to the strong auto acceleration of the reaction rate at conversions higher than $60 \%$ kinetic instabilities may occur in the recycle reactor causing operational problems of the pilot plant. Based on the developed kinetic polymerization model and ideal plug flow behavior of the used static mixers multiple steady states can be predicted. An example is given in Fig.4 for the present reactor configuration and a typical recycle ratio of $\Phi=10$, a wall temperature of $T_{w}=140^{\circ} \mathrm{C}$ and an initiator concentration of $\mathrm{C}_{\mathrm{IO}}=0.05 \mathrm{kmol} / \mathrm{m}^{3}$. At a space time of about $600 \mathrm{~s}$ an abrupt increase of the monomer conversion from $40 \%$ to $94 \%$ occurs. An extinction of the reactor is predicted at ca. $400 \mathrm{~s}$ leading to a conversion drop from $80 \%$ to $20 \%$. The range of the hystereses increases with increasing recycle ratio leading to higher backmixing. Pilot experiments were only conducted under conditions leading to high conversions corresponding to the upper part of the conversion-space time-diagram. Experimental results are summarized in Table 1 . For runs 1 to 4 the wall temperature of the recycle reactor was varied from 149 to $161^{\circ} \mathrm{C}$ and only a small amount of solvent $(7.3 \% \mathrm{wt})$ was added. Under these conditions the acceleration factor is in the order of $30<f_{t}<90$ depending on reaction temperature. A maximal temperature difference between bulk and wall of $15^{\circ} \mathrm{C}$ was observed for run 2 with the highest heat load of more than $1.1 \mathrm{MW} / \mathrm{m}^{3}$. Even under those extreme conditions the reactor could be operated without any problems over several hours. A pressure drop of ca. 40 bar is still acceptable for the present installation designed for a maximal total pressure of 200 bar.

\section{DISCUSSION}

An empirical model describing the gel-effect for the radical polymerization of MMA is proposed to describe quantitatively the polymerization rate as function of time or monomer conversion. The efficency factor is fixed to $f_{a}=0.7$, whereas all other rate constants are used as published (Brandup and Immergut, 1975 and Chiu et al., 1983). Mean molecular mass of of the growing radicals and resulting polymers are obtained by the method of moments (Chiu et al., 1983).

The advantage of the empirical model is the facility to fit the experimentally obtained results even without the quasi steady state assumption. Compared to termination rate constants calculated with published models experimental $k_{t}$ values are one to two orders of magnitudes higher corresponding to a less pronounced increase of the over-all polymerization rate. This may be due to the fact, that published models were developed for a temperature range of about $60^{\circ} \mathrm{C}-90^{\circ} \mathrm{C}$. Therefore it is not astonishing that the model predictions cannot describe the actual experimental results.

To illustrate the ability of our kinetic and models to match experimental observations, calculated results for conversion, mean molecular mass and heterogeneity are compared to experimental ones in Fig. 5 to 7. The difference between calculated and measured conversions is about $\pm 1.5 \%$ whereas the molecular mass and the polydispersity index can be predicted with an accuracy of $\pm 8 \%$.

\section{ACKNOWLEDGEMENT}

The financial support of the "Commission pour l'encouragement de la recherche scientifique, Suisse" Sulzer SA, Winterthur, CH and DSM, Geleen, NL is gratefully acknowledged. 


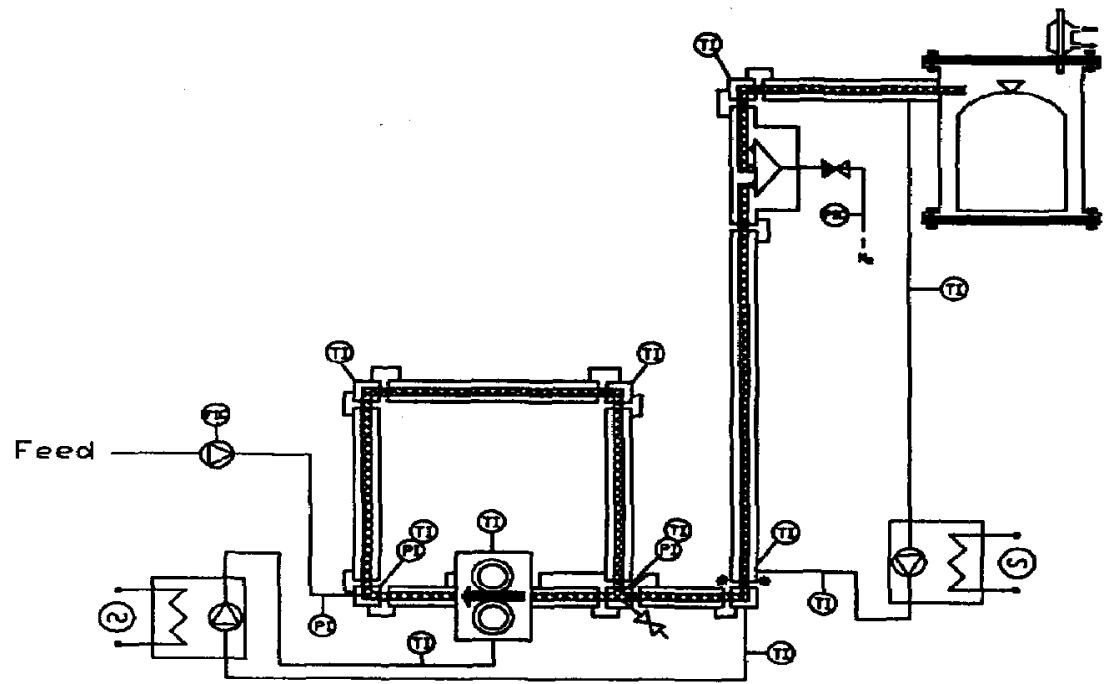

Fig 3 : Experimental Set-up.

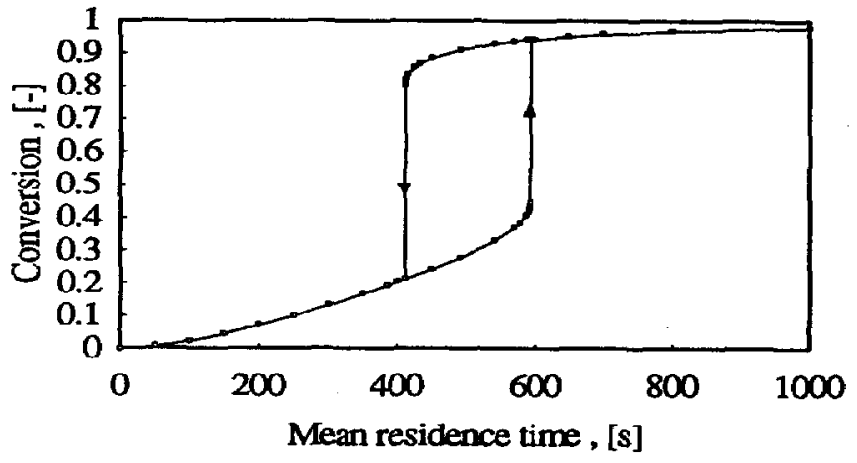

Fig 4 :Stability behavior of the recycle reactor

$$
\begin{aligned}
& \left(\Phi=10, T_{w}=140^{\circ} \mathrm{C}, w_{s o}=0.073\right. \\
& \left.C_{I o}=0.05 \mathrm{M}, V=5.410^{-4} \mathrm{~m}^{3}\right)
\end{aligned}
$$

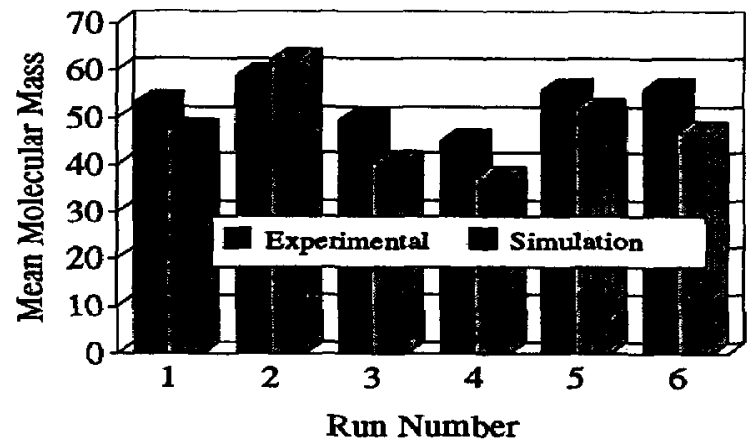

Fig 6 : Experimental and predicted values for the molecular mass in $\mathrm{kg} / \mathrm{mol}$.

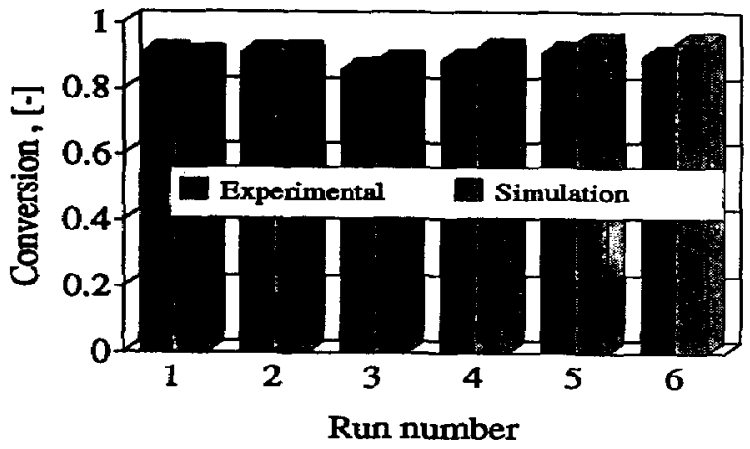

Fig 5 : Experimental and predicted values for the conversion.

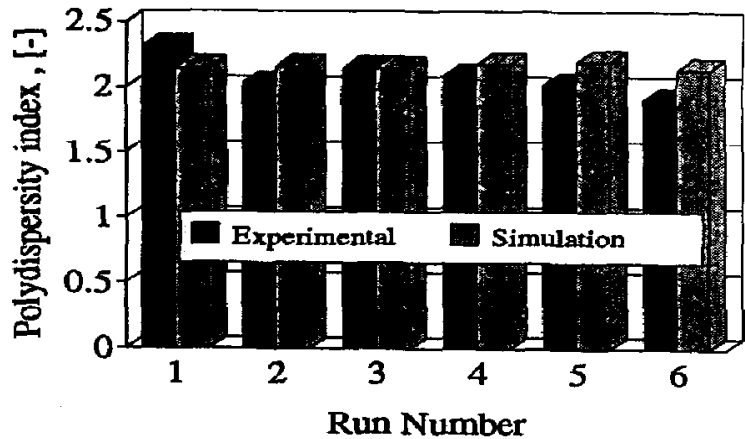

Fig 7 : Experimental and predicted values for the polydispersity index. 
NOMENCLATURE

\section{Latin symbols}

A, B constants, eq. 2

C concentration, $\mathrm{kmol} / \mathrm{m}^{3}$

$c_{p}$ heat capacity, $\mathrm{kJ} /(\mathbf{k g ~ K})$

$d_{t}$ tube diameter, $m$

DTBP di-t-butyl peroxide

f correction function, eq. 1

$f_{a}$ efficiency factor, -

h heat trans.coeff., $\mathrm{kW} /\left(\mathrm{m}^{2} \mathrm{~K}\right)$

$k$ reaction rate constant

$\mathrm{p}$ pressure, $\mathbf{k P a}$

R reaction rate, $\mathrm{kmol} /\left(\mathrm{m}^{3} \mathrm{~s}\right)$
$\mathbf{T}$

$X$ degree of conversion, -

$\mathbf{Z}$ dimensionless reactor length,-

Greek symbols

$\alpha, \beta$ constants eq. 3

$\Delta \mathrm{p}$ pressure drop, $\mathbf{k P a}$

$\Delta \mathrm{H}_{\mathbf{r}}$ reaction enthalpie, $\mathrm{kJ} / \mathrm{kmol}$

$\varphi$ recycle ratio, -

$\phi \quad$ volume fraction -

$\rho$ density, $\mathrm{kg} / \mathrm{m}^{3}$

$\tau$ space time, $s$
Index

d initiator decomposition

eq equilibrium

I ininiator

M monomer

M. radical

P polymer

p propagation

s solvent

t termination

o initial

\section{REFERENCES}

Alberda van Eckenstein, G.O.R.and Tan,Y.Y. 1988, A relatively fast calorimetric method for the determination of initial rate as exemplified by the free radical polymerization of methyl methacrylate in dimethyformamide. Eur.Polym.J. 24, 1073-1075

Balke,S.T.and Hamielec, A.E., 1973, Bulk polymerization of methyl methacrylate. J.Appl.Polymer Sci. $17,905-919$

Brandup, J. and Immergut, E.H., 1975, POlymer Handbook. John Wiley \& Sons, New York

Chiu,W.Y., Carratt, G.M.and Soong, D.S., 1983, A computer model for the gel effect in free-radical polymerization. Macromolecules 16, 348-357

Corpart-Monti, P., 1990, Etude de la cinétique de polymérisation du méthacrylate de méthyle, à haute temperature. Modélisation mathématique. PhD-thesis Strasbourg

Flaschel, E., Nguyen, K.T. and Renken, A., 1985, Improvement of homogeneous tubular reactors operated at low Reynolds number. Proc. 5th European conference on mixing. Würzburg, BHRA. The fluid engineering centre Cranfield, Bedford, 549-554

Juvet, J.E., 1989, Etude de la ségrégation thermique dans un échangeur de chaleur tubulaire. PhD-thesis No 791, Ecole polytechnique fédérale de Lausanne

Louie,B.M., Carratt, G.M. and Soong, D.S., 1985, Modeling the free radical solution and bulk polymerization of methyl methacrylate. J.Appl.Polymer Sci. 30, 3985-4012

Malavasic,T., Osredkar, U., Anzur, I. and Vzovisek, I., 1984, Study of the polymerization of some methacrylic acid esters by differential scanning calorymetry. J. Thermal Analysis 29, 697-700

Marten,F.L. and Hamielec, A.E., 1979, High conversion diffusion contolled polymerization. Am.Chem.Soc.Symp.Ser. 104, 43

Ross,R.T. and Laurence, R.L., 1976, Gel effect and free volume in the bulk polymerization of methy methacrylate. A.I.Ch.E.Symp.Ser.72, 74-79

Schmidt,A.D. and Ray, W.H., 1981, The dynamic behavior of continuous polymerization reactors-I. Chem.Eng.Sci. 36, 1401-1410

Stickler, M., Panke, D. and Hamielec, A.E., 1984. Polymerization of methyle methacrylate up to high degrees of conversion: Experimentell investigation of the diffusion contolled polymerization. J.Polymer Sci. 22, 2243-2253

Sulzer, 1991a, Polymerization Plants. Sulzer AG, Winterthur, Switzerland, No: 22.00.06.40-V. 91-50

Sulzer, 1991b, Mixing process equipment. Sulzer AG, Winterthur, Switzerland, No: 23.27.06.20-V.91100 